\title{
Deficiencies of fibrillin and decorin in fibroblast cultures of a patient with neonatal Marfan syndrome
}

\author{
A Superti-Furga, M Raghunath, P J Willems
}

\begin{abstract}
Changes in the structure and metabolism of fibrillin, a microfibril associated protein, can result in classical Marfan syndrome, and reduced expression of decorin, a small extracellular chondroitin sulphate/dermatan sulphate proteoglycan, has been observed in fibroblasts of a patient with neonatally lethal Marfan syndrome. We have studied the synthesis of fibrillin and decorin in cultured fibroblasts of a further sporadic patient with neonatally lethal Marfan syndrome. Fibrillin immunoreactivity in the extracellular matrix of the patient's fibroblasts was markedly reduced, and the fibrillar pattern was absent, in spite of normal amounts of fibrillin mRNA. Decorin mRNA, synthesis, and immunoreactivity in the matrix were also reduced. The results indicate involvement of both fibrillin and decorin in the pathogenesis of neonatal Marfan syndrome in this patient, but do not indicate which is the primary defect. We speculate, however, that a structural defect of fibrillin leads to diminished incorporation of the protein into the extracellular matrix, and that underexpression of decorin is secondary to the primary fibrillin defect. Combined deficiency of fibrillin and decorin may be the cause of the severe clinical phenotype.

( $\mathcal{F}$ Med Genet 1992;29:875-8)
\end{abstract}

Family linkage analysis and immunofluorescence studies in patients with dominantly inherited Marfan syndrome (MS) have recently converged to show that the MS locus is on chromosome $15^{1}$ and is identical to the gene of fibrillin, a microfibril associated protein with molecular weight of $350 \mathrm{kDa} .^{2-4}$ The pathogenetic role of fibrillin has been confirmed by the identification of mutations in the fibrillin gene in patients with classical $\mathrm{MS}^{56}$ (L Peltonen-Palotie, personal communication). Fibroblast cultures of MS patients show subtle changes in the biosynthesis and extracellular matrix deposition of fibrillin. ${ }^{7}$ A second fibrillin gene on chromosome 5 has been linked to a phenotypically similar but distinct disorder, congenital contractural arachnodactyly (CCA $)^{3}$; in this disorder, no cardiovascular or ocular anomalies are found.

The so-called neonatal Marfan syndrome has features of classical Marfan syndrome, such as body disproportion and heart valve insufficiency, but also additional features such as cutis laxa, joint contractures, muscle hypoplasia, crumpled ears, congenital anomalies of the eye, and pulmonary emphysema. ${ }^{8}$ The molecular relationship of neonatal Marfan syndrome to classical MS and to CCA is not yet clear. In cells from one infant, deficiency of decorin, a small proteoglycan of the extracellular matrix, was observed, ${ }^{9}$ while in another case, deficiency of laminin and fibronectin was reported. ${ }^{10}$ In neither case was fibrillin studied. The present study was undertaken in order to investigate the involvement of fibrillin and decorin in the pathogenesis of neonatally lethal Marfan syndrome in another patient with this rare disorder.

\section{Case report}

A detailed description of the clinical and pathological findings has been reported earlier in the Journal. ${ }^{8}$ Briefly, the patient was the first child of unrelated parents with no signs of Marfan syndrome except for mitral valve prolapse in the mother. He died 20 hours after birth of cardiac failure. Length was $55 \mathrm{~cm}$ and weight was $3400 \mathrm{~g}$. He had arachnodactyly, flexion contractures of several major joints, crumpled ears, loose skin with absence of subcutaneous fat, and hypoplasia of the calf muscles. Pathological examination showed biventricular enlargement of the heart with valve insufficiency, and presence of congenital anomalies of the iris and ciliary processes without lens dislocation. ${ }^{8}$

\section{Methods}

Cultured skin fibroblasts of the patient, of neonatal controls, and of adult MS patients were seeded at high density in Lab-Tek chamber slides (Nunc) and incubated for 48 hours. Fibrillin deposition in the extracellular matrix of fibroblasts grown on the slides was studied using a fibrillin specific monoclonal antibody (Mab F2, ${ }^{2}$ a gift of Dr M Godfrey, Omaha, Nebraska) and a goat anti-mouse Texas Red conjugate (Dianova). The same slides were stained with a polyclonal rabbit antiserum raised against the core protein of decorin (a gift of Dr Hans Kresse, Münster, FRG) and a swine anti-rabbit FITC conjugate (DAKO).

To study decorin production, fibroblast cultures were labelled with either ${ }^{35} \mathrm{~S}$-methionine and cysteine (TranSlabel, Flow) or carrier free ${ }^{35} \mathrm{~S}$-sodium sulphate (Flow) for 16 hours, and the medium was applied to a DEAE-Trisacryl 
ion exchange column (Serva) to isolate the proteoglycans, essentially as described by Glössl et al. ${ }^{11}$ The proteoglycans were concentrated using Centricon-30 spun dialysis columns (Amicon), separated by electrophoresis on sodium dodecylsulphate-polyacrylamide 4 $15 \%$ gradient gels, and visualised by autoradiofluorography. Aliquots of the proteoglycan preparations were treated with chondroitinase ABC (Seikagaku) before electrophoresis. Decorin and biglycan were immunoprecipitated from patient's and control medium using specific antisera and protein A-Sepharose (Pharmacia) and electrophoresed.

Total fibroblast RNA from the proband and from controls was extracted by the guanidinium isothiocyanate-caesium chloride method, separated on agarose gels, blotted onto nylon membranes, and hybridised to ${ }^{32} \mathrm{P}$ labelled decorin and biglycan cDNA probes (obtained by PCR amplification of control fibroblast cDNA using primers designed from the published sequences ${ }^{1213}$ ) and to a fibrillin cDNA probe $\left(\mathrm{MF}-13^{3}\right)$.

Structure and synthesis of collagens I, III, and $\mathrm{V}$ in the proband's cells had been previously studied in our laboratory and found to be normal. ${ }^{8}$

\section{Results}

\section{FIBRILLIN}

Fibrillin immunoreactivity in the extracellular matrix of neonatal control fibroblast cultures was present as a reticular pattern (fig 1, left). In seven adult $M S$ cell strains the intensity was reduced, but the fibrillar pattern was still present (not shown). In the neonatal MS patient, immunoreactivity was markedly reduced, and fibrils could barely be identified (fig 1); fibrillin immunoreactivity was limited to small clumps. There was no evidence of intracellular storage of fibrillin. Northern blotting showed that the concentration of fibrillin mRNA in the proband's cells was similar to that in control cells (fig 2).

DECORIN

There was a fine web of decorin immunoreactivity in the extracellular matrix of control cultures (fig 1), but this diffuse web was not seen in the neonatal MS patient (fig 1, right). The clumps previously observed to react with the fibrillin antibody were also positive for decorin, indicating at least partial co-localisation of these two molecules in the patient. Biochemical studies showed that the patient's cells secreted less decorin than control cells (fig 3); decorin secretion was somewhat variable between repeated labelling experiments, but consistently lower than that of control cells. Secretion of biglycan, a related proteoglycan, was normal, indicating that there was no general defect in proteoglycan biosynthesis. Immunoprecipitation of decorin and biglycan confirmed decorin deficiency in the patient's medium (not shown); glycosaminoglycan free core protein was not detected. ${ }^{14}$ Reduced synthesis of decorin was associated with a marked reduction in decorin mRNA as shown by northern blotting (fig 2); levels of biglycan mRNA were slightly increased (data not shown). Cells from three adult MS patients secreted normal amounts of decorin (not shown).

\section{Discussion}

The study was undertaken in order to ascertain whether fibrillin or decorin or both were

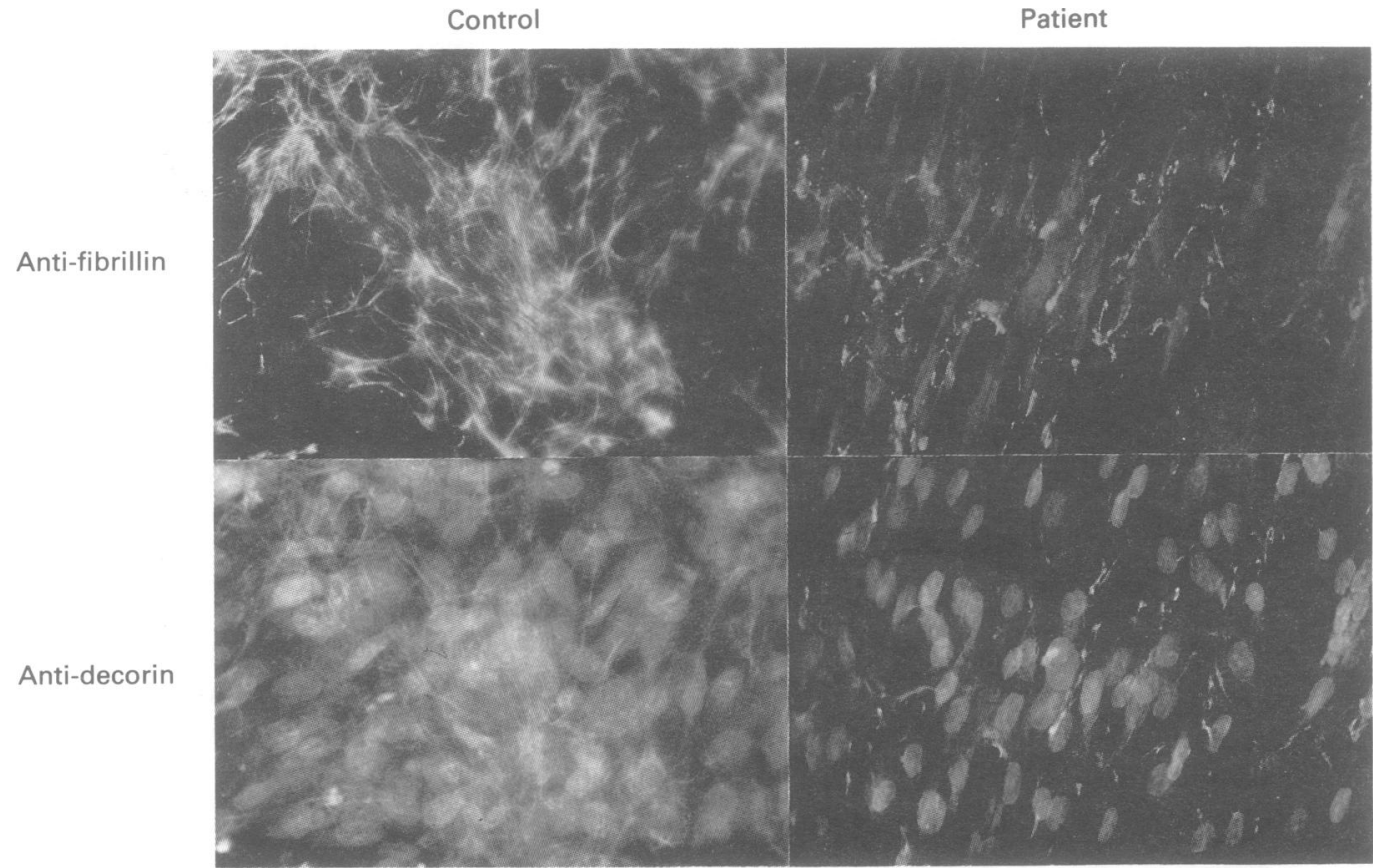

Figure 1 Double immunofluorescence study of cells of a neonatal control (left) and of the patient (right) using a monoclonal antibody against fibrillin (top) and an antiserum against decorin (bottom). The same fields are shown in each column. In the control, fibrillin positive fibrils are clearly visible; in the patient, immunoreactivity is reduced in intensity and confined to small clumps. Decorin is present in the control both in fibrils and as a diffuse layer; in the patient, it is quantitatively reduced and localised to the same structures which were positive for fibrillin. 


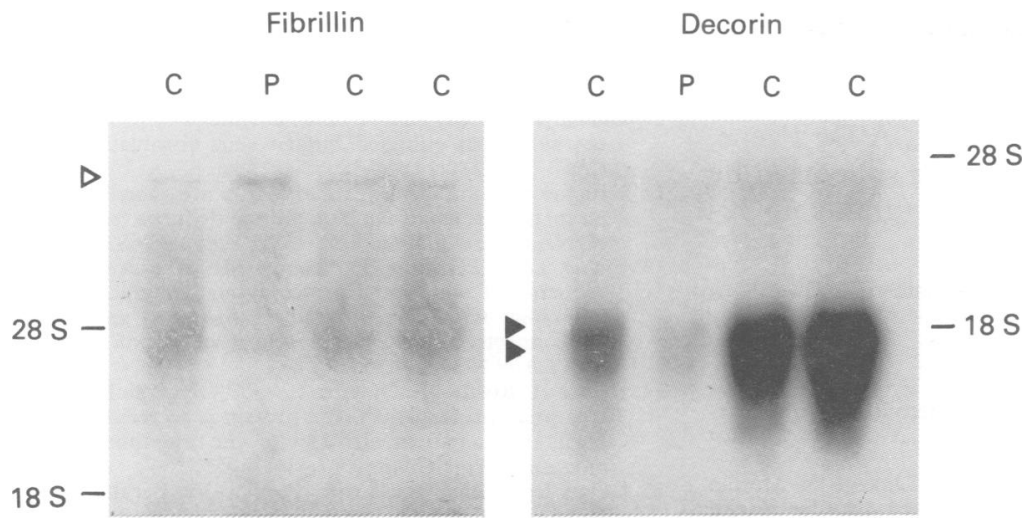

Figure 2 Northern blot analysis of total RNA from control $(C)$ and the patient's (P) fibroblasts probed with a fibrillin cDNA (left) or decorin cDNA probe (right). Fibrillin mRNA (open arrowhead) is normal or perhaps slightly increased in the patient, while decorin mRNA (filled arrowheads, two populations differing by their polyadenylation site) is decreased; note, however, that there are differences in decorin $m R N A$ concentration between the controls also.

involved in the pathogenesis of neonatal Marfan syndrome. The results indicate an involvement of both, but do not discriminate clearly which is the primary defect.

Reduced fibrillin immunoreactivity and abnormal fibril shape were found, together with normal amounts of fibrillin mRNA. This would be compatible with a structural defect in the fibrillin protein, ${ }^{5}$ leading to its diminished incorporation into the extracellular matrix, and would suggest that a de novo dominant mutation is responsible for the phenotype. This hypothesis remains to be proven by the identification of a mutation in the fibrillin gene.

Because of the previous report of underexpression of decorin in fibroblasts from a neonatally lethal Marfan syndrome patient, ${ }^{9}$ decorin synthesis was also studied in our patient. Decorin mRNA, biosynthesis, and immunoreactivity were indeed found to be

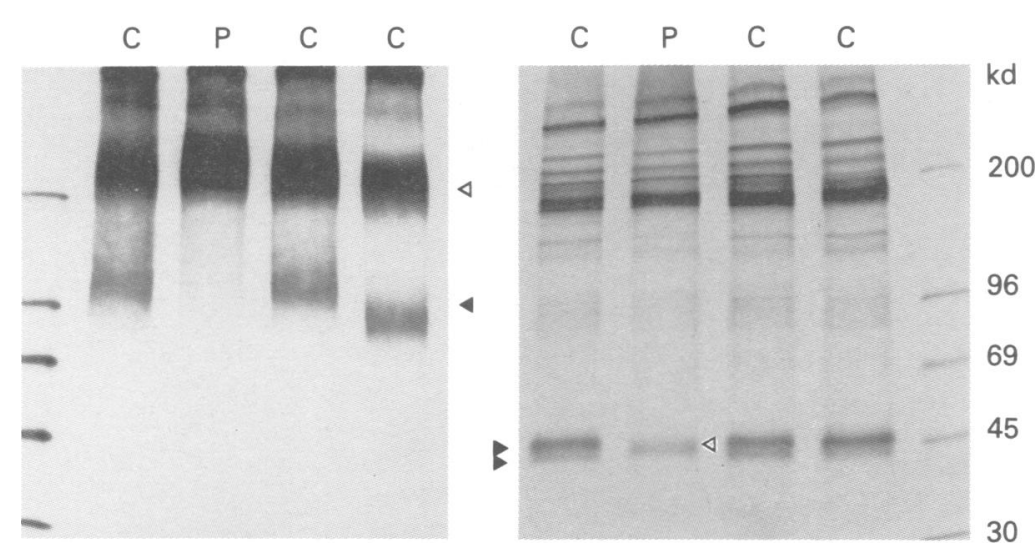

Figure 3 Proteoglycans in the culture medium of fibroblasts from the patient $(P)$ and from controls (C). (Left) After incubating fibroblasts with ${ }^{35} S$-sodium sulphate to label the glycosaminoglycan side chains, proteoglycans were purified and directly separated by electrophoresis as described in the text. The open arrowhead points at biglycan, the filled arrowhead at decorin. Slight differences in the migration of decorin are the result of different lengths of the glycosaminoglycan side chain. Note deficiency of decorin in the patient. (Right) After incubating fibroblasts with ${ }^{35} S$-methionine to label the core proteins, proteoglycans were purified, treated with chondroitinase $A B C$ (see text) to remove side chains, and separated by electrophoresis. Molecular weight standards are indicated on the left. Decorin and biglycan core proteins ( $329 v 331$ amino acid residues) migrate similarly, as shown by selective immunoprecipitation (not shown). The filled arrowheads point at the two decorin core proteins (which differ only by the number of $N$ linked glycosides), the open arrowhead at the biglycan core protein. In the patient, only the biglycan core protein is evident. reduced. Decorin is a small proteoglycan composed of a core protein of 329 amino acids and a single dermatan sulphate/chondroitin sulphate side chain of approximately $35 \mathrm{kDa}$ and two or three $\mathrm{N}$ linked glycosides. ${ }^{112}$ It is associated with the surface of collagen fibrils (and 'decorates' them, hence the name). Reduced decorin expression had been previously found in another patient with severe neonatal MS, ${ }^{9}$ while a defect in glycosylation of decorin has been described in a boy with osteoporosis, lax skin, and progeroid features. ${ }^{14}$ Recently, underexpression of decorin has been found in fibroblasts of several patients affected with the neonatal pseudohydrocephalic progeroid syndrome (WiedemannRautenstrauch syndrome), another condition featuring among other changes thin and lax skin with paucity of subcutaneous fat ${ }^{1516}(\mathrm{H}$ Kresse, personal communication).

The significance of reduced expression of decorin in cells from our patient is not clear; specifically, it is not possible to distinguish between decorin gene mutations and a secondary, regulatory phenomenon. Although a primary decorin gene mutation cannot be ruled out, we favour the second hypothesis for the following reasons. Firstly, decorin expression was constantly below normal but there was significant variation between single experiments. Secondly, decorin underexpression has been observed in fibroblasts of patients with Wiedemann-Rautenstrauch syndrome, a possibly related, but clearly distinct, disorder. Lastly, it is known that decorin expression in fibroblasts can be regulated by a variety of cytokines, for example, by TGF- $\beta .^{81718}$ Interestingly, TGF- $\beta$ can be bound and neutralised by decorin itself. ${ }^{1920}$ Since fibrillin contains a putative TGF binding region, ${ }^{4}$ it is conceivable that alterations in the extracellular matrix or, more specifically, in the biosynthesis or degradation of fibrillin may perturb feedback mechanisms controlling decorin expression. Whichever the mechanism, decorin underexpression may contribute to the severity of the clinical picture, as we have not observed it in cells from adult MS patients with abnormal fibrillin immunostaining patterns, but have recently found combined fibrillin and decorin deficiency in the extracellular matrix of a new patient with neonatally lethal $M S^{21}$

We conclude that the neonatally lethal Marfan syndrome in the proband was associated with defective deposition of fibrillin and decorin in the extracellular matrix of cultured fibroblasts. Although the data do not allow discrimination of the primary defect, we suggest that diminished fibrillin incorporation is caused by a structural mutation, since fibrillin mRNA levels are normal. Reduced synthesis of decorin, associated with low mRNA levels, may be a secondary phenomenon, although a decorin gene mutation cannot be ruled out. It is conceivable that combined fibrillin and decorin deficiency may be responsible for the severe clinical phenotype.

We thank Dr $\mathrm{H}$ Kresse for the decorin and biglycan antisera, for technical advice, and for 
sharing unpublished information; Dr M Godfrey for the fibrillin antibody; and Dr F Ramirez for the fibrillin cDNA probe. We are also indebted to Drs I Buntinx, A De Paepe, and L Peltonen. This work was supported by the Swiss National Foundation (grants no 3230148.90 and 32-27884.89).

1 Kainulainen $\mathrm{K}$, Pulkkinen L, Savolainen A, Kaitila I, Peltonen L. Location on chromosome 15 of the gene defect causing

2 Hollister DW, Godfrey M, Sakai LY, Pyeritz RE. Immunohistologic abnormalities of the microfibrillar-fiber system in the Marfan syndrome. N Engl $f$ Med tem in the

3 Lee B, Godfrey $M$, Vitale E, et al. Linkage of Marfan syndrome and a phenotypically related disorder to two different fibrillin genes. Nature 1991;352:330-4.

4 Maslen CL, Corson GM, Maddox BK, Glanville RW, Sakai LY. Partial sequence of a candidate gene for the Marfan syndrome. Nature 1991;352:334-7.

5 Dietz HC, Cutting GR, Pyeritz RE, et al. Marfan syndrome caused by a recurrent de novo missense mutation in the fibrillin gene. Nature 1991;352:337-9.

6 Dietz HC, Pyeritz RE, Sakai LY, Francomano CA, Cutting GR. Variability of cellular and clinical phenotype in the Marfan syndrome: three novel mutations in the fibrillin gene. Pediatr Res 1992;31:132A.

7 McGookey-Milewicz D, Pyeritz RE, Crawford ES, Byers PH. Marfan syndrome: defective synthesis, secretion, and extracellular matrix formation of fibrillin by cultured extracellular matrix formation of fibrillin by
dermal fibroblasts. $f$ Clin Invest 1992;89:79-86.

8 Buntinx IM, Willems PJ, Spitaels SE, Van Reempts PJ, De Paepe AM, Dumon JE. Neonatal Marfan syndrome with congenital arachnodactyly, flexion contractures, and congenital arachnodactyly, flexion contractures, and severe cardiac

9 Pulkkinen L, Kainulainen K, Krusius T, et al. Deficient expression of the gene coding for decorin in a lethal form of Marfan syndrome. $f$ Biol Chem 1990;265:17780-5.
10 Bonneau D, Huret JL, Godeau G, et al. Recurrent $\mathrm{ctb}(7)(\mathrm{q} 31.3)$ and possible laminin involvement in a neonatal cutis laxa with a Marfan phenotype. Hum Genet natal cutis laxa

11 Glössl J, Beck M, Kresse H. Biosynthesis of proteodermatan sulfate in cultured human skin fibroblasts. $\mathcal{f}$ Biol Chem 1984:259:14144-50.

12 Krusius T, Ruoslahti E. Primary structure of an extracellular matrix proteoglycan core protein deduced from cloned cDNA. Proc Natl Acad Sci USA 1986;83:7683-7.

13 Fisher LW, Termine JD, Young MF. Deduced protein sequence of bone small proteoglycan I (biglycan) shows homology with proteoglycan II (decorin) and several nonconnective tissue proteins in a variety of species. $\mathcal{f}$ Biol Chem 1989;264:4571-6.

14 Kresse H, Rosthoj S, Quentin E, et al. Glycosaminoglycanfree small proteoglycan core protein is secreted by fibroblasts from a patient with a syndrome resembling progeroid. Am f Hum Genet 1987;41:436-53.

15 Devos EA, Leroy JG, Frijn JP, Van den Berghe H. The Wiedemann-Rautenstrauch or neonatal progeroid syndrome. Eur f Pediatr 1981;136:245-8.

16 Kresse H, Quentin-Hoffmann E, Beavan LA. Proteoglycans: astonishing complexity of structure, function and involvement in genetic disease. Abstract Book of the Annual Meeting of the European Society for Pediatric Research, Zurich, 1-4 September 1991, abstract no 96.

17 Breuer B, Schmidt G, Kresse $H$. Non-uniform influence of transforming growth factor- $\beta$ on the biosynthesis of different forms of small chondroitinsulphate/dermatan sulphate proteoglycan. Biochem $\mathcal{f} 1990 ; 269: 551-4$

18 Kähäri VM, Larjava $\mathrm{H}$, Uitto J. Differential regulation of extracellular matrix proteoglycan (PG) gene expression. $\mathcal{f}$ Biol Chem 1991;266:10608-15.

19 Yamaguchi Y, Mann DM, Ruoslahti E. Negative regulation of transforming growth factor- $\beta$ by the proteoglycan decorin. Nature 1990;346:281-4.

20 Ruoslahti E, Yamaguchi Y. Proteoglycans as modulators of growth factor activities. Cell 1991;64:867-9. 21 Raghunath $M$, Superti-Furga A, Godfrey $M$, Steinmann $A$. in neonatal Marfan syndrome fibroblasts. Hum Genet (in press). 